\title{
Systèmes technologiques et régimes de risques industriels
}

\author{
Technological Systems and Evolution of Industrial Risks
}

\author{
Smaïl Aït-el-hadj ${ }^{1}$ \\ ${ }^{1}$ Université de Lyon, ITECH, France, smail.aitelhadj@itech.fr
}

\begin{abstract}
RÉSUMÉ. L'objet de cet article est de montrer la relativité des risques industriels par rapport aux trois systèmes technologiques qui se sont succédé durant l'histoire industrielle. A des risques touchant principalement les industries minières et les machines à vapeur dans le premier système technologique, risques limités au site et au personnel, succèdent dans le deuxième système technologique les grands accidents chimiques et nucléaires, formalisés sous le concept de risque technologique majeur. Les risques industriels du troisième système technologique, sont marqués par leur échelle et générés par leur complexité telle que le risque cybernétique, et aussi par l'accumulation historique des nuisances de toute l'histoire industrielle pour le risque environnemental global. Cette dernière configuration modifie l'influence du risque industriel sur la trajectoire même de l'évolution technologique.

ABSTRACT. The aim of this article is to show the relativity of industrial risks regarding the three technological systems that occurred successively in industrial history. The risks which mainly affect the mining industry and steam engines in the first technological system, limited to on-site risks and risks for personnel, were superseded in the second technological system by enormous chemical and nuclear accidents, formalized by the concept of major technological risk. The industrial risks of the third technological system were characterized by their scale and generated by their complexity, such as cybernetic risk, and also by the historical accumulation of nuisances from the industrial history for global environmental risk. This last configuration modifies the influence of industrial risks on the actual course of the technological evolution.

MOTS CLÉS. systèmes technologiques, dynamique technologique, innovation, risques industriels, catastrophes technologiques.

KEY WORDS. technological systems, technological dynamics, innovation, industrial risks, technological catastrophes.
\end{abstract}

\section{Introduction}

La modélisation de l'histoire industrielle à travers la succession de systèmes technologiques est un bon guide pour la compréhension de la genèse et de l'évolution des risques industriels aussi bien dans leur nature technique que dans leur ampleur ou que dans leur intégration dans les modes de pilotage technique et de régulation industrielle ce que nous appelons régimes de risques industriels. Les risques industriels n'ont pas été d'une nature et d'une ampleur constante au cours de l'histoire, mais ils ont bien été déterminés par le type de technologies mises en œuvre ainsi que par la complexité et l'échelle d'activité qu'elles ont permis ; ceci parce que la nature et les formes des systèmes technologiques induisent et expliquent des types de risques et de catastrophes spécifiques, mais aussi parce que la réalisation et la prise en compte ultérieure de ces risques ont un effet en retour sur l'itinéraire voire les limites de ces systèmes techniques, contribuant à des processus d'innovation, voire à des situations d'obsolescence'.

Dans cette exploration, nous parlons génériquement de risques, une classe d'évènements négatifs en général entendus comme probables, mais nous allons souvent nous référer à des accidents et des catastrophes effectives, évènements réalisés, dont il est indispensable de rendre compte pour comprendre les risques dans leur dimension d'évènement et de dommages (DAUPHINE, PROVITOLO, 2013).

1. Boucle de rétroaction qui est en voie de transformer la théorie même de la systémique technologique par l'intégration de l'anticipation et de la maîtrise des risques dans leurs dynamiques d'évolution déjà bien élaborée dans : Engineering systems, (DE WECK, 2011). 
Nous allons explorer dans cet article comment le développement de systèmes technologiques, de nature différentes mais aussi d'une complexité et de puissance de transformation croissantes, va conduire à l'apparition de la notion de risque industriel, au moment de la première industrialisation anglaise (FRESSOZ, 2012) et aussi comment cette évolution va faire apparaitre des types de risques nouveaux, des risques chimiques du deuxième système technologique, aux cyber-risques et aux risques environnementaux, issus des technologies nouvelles apparues dans le système technologique contemporain. Ces risques vont non seulement se renouveler dans leur nature mais amplifier leur échelle de conséquence, du risque industriel de site, à un risque artificiel universel, dans un mouvement d'élargissement et de complexification.

Nous montrerons ce processus à travers un certain nombre de risques, des accidents et catastrophes auxquels elles ont donné lieu, emblématiques de chacun des systèmes technologiques sans pouvoir rendre compte, dans notre présentation limitée, de l'ensemble des formes de risques de ces systèmes.

\section{Le cadre permanent de l'innovation : le système technologique}

Le cadre de la modélisation de la dynamique récente de l'innovation est la théorie des systèmes technologiques, dite aussi systémique technologique (AÏT-EL-HADJ, 2014). Nous allons présenter ce cadre d'analyse à partir de trois mécanismes le constituant: les caractéristiques et l'évolution d'un système technologique en tant que structure, la modélisation du cycle de vie des technologies, la dialectique interne au mouvement technologique à partir de l'interaction procédé/structure/fonction qui permet d'identifier et de comprendre la dominante du mode d'innovation à un moment donné de l'évolution du système technologique, mais aussi le mécanisme de déploiement d'un système technologique dans la société (AÏT-EL-HADJ, 2015).

\subsection{Qu'est-ce qu'un système technologique?}

La systémique technologique est fondée sur la notion originelle de "système technique » formalisée par Bertrand Gille (1978). Qu'est-ce alors qu'un système technique ? C'est le modèle qui va rendre compte de cette relation fondatrice: «dans une période donnée et à des degrés divers, toutes les technologies sont interdépendantes et cohérentes entre elles.» (GILLE, 1978). Ainsi toute la technologie d'une période va être structurée par une solidarité générale qui va diffuser, unifier, les technologies du système, en même temps qu'elle va constituer le puissant moyen de génération et de diffusion des innovations. En effet, chaque changement technologique en un point du système, va constituer une incitation au changement par les nouvelles possibilités qu'il engendre, ou constituer une contrainte au changement par les requêtes quantitatives et qualitatives, nécessaires à son développement qu'il adresse à son système d'interrelations technologiques (HUGHES, 1987).

Ainsi le système technologique, par son jeu de relations structurelles, va constituer une base permanente de changement technologique en diffusant, relayant et amplifiant toute perturbation de changement technique.

Le système technologique constitue aussi un système hiérarchique par l'ampleur de diffusion de chacune des technologies. L'ensemble des technologies d'un système procède d'un petit nombre de moyens centraux de transformation de la matière/énergie/information qu'il est convenu d'appeler technologies génériques. C'est cette intégration hiérarchisée des technologies qui génère un ensemble unifié, certains disent un "paradigme technologique » (DOSI, 1982), qui va explorer tout son champ de possibles, avant de s'épuiser et de laisser la place à un nouveau système fondé sur un groupe de nouvelles technologies génériques, un nouveau paradigme structurant un nouveau système technologique. Cette dynamique est à l'origine de cette discontinuité du mouvement technique à travers la succession des systèmes technologiques. 


\subsection{La succession historique des systèmes technologiques}

Ce modèle permet d'interpréter l'histoire technologique et la dynamique de longue durée de l'innovation comme une succession de systèmes technologiques. On a pu déjà construire une formalisation de la technologie de périodes relativement anciennes sous la forme, plus ou moins achevée, de systèmes technologiques. Ceci déjà pour la préhistoire, mais aussi pour un système technologique antique, ainsi que pour un grand système, novateur quoique fondé sur des technologies génériques traditionnelles telles que l'énergie animale, hydraulique et éolienne, sur le bois, la pierre et les fibres naturelles, qui partant d'une révolution technologique du XII siècle va s'achever à la fin du XVIII ${ }^{\mathrm{e}}$ siècle pour déboucher sur la révolution industrielle anglaise. Celle-ci va mettre en place le premier système technologique industriel fondé sur la sidérurgie du fer, l'énergie de vapeur alternative, la mécanisation du textile qui ont constitué ses technologies génériques et fourni les bases de la première transformation industrielle de grande ampleur.

Ce système a montré des signes d'épuisement à la fin des années 1880 , pour donner naissance à un nouveau système technologique, dit deuxième système technologique, fondé lui sur l'acier et les alliages, la mécanique, l'électricité, les énergies fossiles, qui va avoir des effets de transformation et d'innovation encore plus considérables que le système précédent, comme le montre bien l'historien David Landes (1975).

Le troisième système technologique, qui est en fait le troisième système technologique de l'ère industrielle, est apparu à la fin des années 1970, à la suite de l'épuisement des technologies génériques du deuxième système technologique. Son effondrement est aussi lié à la crise énergétique des années 1970 et à la crise de la relation de ce système avec son environnement (MENSCH, 1980).

En réponse à cette crise et en dépassement de cet épuisement technologique le nouveau système s'est constitué sur de nouvelles technologies génériques apparues au même moment et porteuses d'un grand potentiel de développement, telles que la microélectronique, la biologie à composante génétique, un marqueur de ces apparitions ayant été en 1975 et 1976, l'attribution des prix Nobel aux inventeurs du micro-processeur et des techniques d'intervention sur l'ADN.

Ce faisceau de technologies génériques va être complété par les nouvelles problématiques de matériaux centrées sur les composites et plus généralement sur ce qu'A.Y. Portnoff a appelé «l'hyperchoix des matériaux » (CPE, 1983). Enfin, les technologies génériques énergétiques, évoluent vers la diversité en réponse à la crise de l'énergie en partie à l'origine de cette grande mutation technologique.

Ce système technologique $\mathrm{a}$, depuis environ 35 ans, connu plusieurs vagues. La première a été largement centrée sur la micro-électronique et le déploiement du micro-processeur, elle s'est déroulée jusqu'aux années 1990. Un mouvement de relais a été pris, par les technologies de l'information en réseau et l'exploitation des possibilités de la numérisation généralisée.

S'ouvre avec cette période moderne la succession de trois systèmes technologiques, dont les deux premiers se sont respectivement développés sur une période approximative de cent ans et le dernier qui nous occupe ici est encore en cours de déploiement. C'est de ces trois systèmes technologiques successifs que nous allons établir la nature et la spécificité des régimes de risques industriels. 
2. Les risques technologiques dans les sociétés traditionnelles et première société industrielle : le risque catastrophique localisé

\subsection{Les risques technologiques dans la société traditionnelle}

L'accident technique est consubstantiel à l'activité humaine et dès la société antique puis médiévale l'activité technique des métiers et des chantiers a donné lieu à des accidents divers qui ont entrainé des dégâts et pertes mais le plus souvent à l'échelle individuelle ou de petits groupes : accident du travail individuel, accident de chantier et effondrement d'édifice en construction. Les plus importants touchant dans toute cette période les chantiers, déjà de grande échelle, de la construction et du génie civil antique et médiéval.

$\mathrm{C}^{\prime}$ est $\mathrm{au} \mathrm{XVII}^{\mathrm{e}}$ et au XVIII ${ }^{\mathrm{e}}$ siècle qu'apparaît le premier grand risque industriel massif et récurrent, celui lié à la poudre à canon, qui avait donné lieu à la constitution de stocks, souvent près des villes. En 1645 le tiers de la ville de Boston est détruit par l'explosion d'un stock de poudre, en 1654 c'est une grande partie de la ville de Delft en Hollande qui sera détruite par une explosion de poudrière, entrainant plusieurs centaines de morts, accident qui sera popularisé par un tableau de Vermeer. Le 31 août 1794 l'explosion de la poudrerie de Grenelle va entrainer plus de 1000 morts, provoquant un des accidents industriels les plus meurtriers de l'histoire en France (LE ROUX, 2011).

Avec l'usage à grande échelle des explosifs pour les munitions, notamment dans les guerres ultérieures, ces catastrophes vont se perpétuer. Ceci, jusqu'à une gigantesque catastrophe qui en est le sommet, le 6 décembre 1917, dans le port d'Halifax, en Nouvelle-Écosse (Canada), l'explosion du navire Mont-Blanc survient à quai, lors du chargement de 9000 tonnes de munitions hautement explosives. 2000 personnes sont tuées et 9000 sont blessées, alors qu'une partie de la ville est soufflée par l'explosion. De nombreuses explosions accidentelles de munitions interviendront pendant la Seconde Guerre mondiale, mais les poudres et explosifs cesseront dès le XIX ${ }^{\mathrm{e}}$ siècle de constituer la principale source de risque industriel.

\subsection{L'apparition de nouveaux types de risque avec la révolution industrielle anglaise}

Ces bases d'accidents technologiques vont se multiplier et se pérenniser avec la première révolution technologique anglaise celle-ci va mettre en œuvre des nouvelles technologies dont la puissance opératoire va comporter, comme la poudre à canon, dans le siècle précédent un facteur de puissance destructrice décuplée. Ces technologies comme la machine à vapeur à pression, et le gaz d'éclairage en particulier vont se disséminer, non seulement dans des usages industriels massifs mais aussi dans les transports, ferroviaire et maritime, et dans l'utilisation urbaine.

Mais aussi avec l'élargissement et l'approfondissement d'activités traditionnelles telles que l'activité minière. Il ne s'agit pas là du développement d'une technologie à puissance renforcée mais de la mise en œuvre d'une activité, déjà en soi dangereuse mais à des échelles et à des profondeurs telles qu'elle va générer le risque de la survenue répétitive de nouvelles véritables catastrophes.

\subsubsection{L'accident minier}

Concentré dans les mines de charbon, l'accident minier est, dans toute la période du premier système technologique industriel et même dans celui qui lui succédera après 1880 , non seulement un type majeur d'accident industriel collectif mais l'origine d'un grand nombre d'accidents individuels causant par exemple en 1850 plus de 19 tués par million de tonnes de charbon extrait, soit 2500 morts par an en moyenne dans cette période (Amoudru,1983). De plus dans chacun des grands pays charbonniers surviennent régulièrement des catastrophes, par explosions dues à des coups de grisous, « coups de poussière » ou incendies. Ainsi en Grande-Bretagne, entre 1835 et 1850, on ne compte pas moins de 645 explosions, et on dénombre 31 catastrophes minières dans la décennie 1850-1860 ayant fait 1270 morts. Les trois décennies ultérieures jusqu'en 1890, connaitront une moyenne d'une 
trentaine de catastrophes par décennie, avec un pic de mortalité de plus de 2000 morts dans la période 1870-1880. Cette fréquence se réduit considérablement à partir de 1890, où la fréquence des accidents est divisée par 3 et celle de la mortalité par 2. Alors que l'Allemagne entrée plus tard dans la grande industrie charbonnière connait plus de 70 catastrophes de 1891 à 1900. Pourtant la plus grande catastrophe minière européenne, la catastrophe de Courrières en France, qui fait en un seul accident plus de 1000 morts, se produit en 1906, alors que, dans cette période, la France connaissait une inflexion positive parallèle à la Grande-Bretagne.

Cette diminution relativement rapide de la sécurité dans les mines de charbon est significative du changement de système technologique intervenu à la fin du XIX ${ }^{\mathrm{e}}$ siècle. Les grands accidents miniers étaient dus à au moins deux types de phénomènes physico-chimiques : le coup de grisou, dégagement et accumulation de méthane dans les galeries, explosant au contact d'une flamme nue et le «coup de poussière » engendré par une atmosphère détonante créée par une certaine proposition de poussière de charbon dans l'air ambiant, le mélange se comportant comme un gaz inflammable. C'est par l'accumulation d'expérience liée à ces catastrophes que furent mis au point empiriquement des dispositifs de sécurité comme les lampes de sécurité, et plus tard la mise en sécurité des circuits électriques. Surtout la compréhension scientifique de ces phénomènes physico-chimique va permettre de concevoir et de mettre en place des dispositifs de sécurité préventifs et curatifs soutenus par les technologies nouvelles, notamment l'électricité et plus tard l'électronique : dispositifs d'aération forcée, grisoumètres, matériaux de machines ininflammables... (AMOUDRU, 1983)

\subsubsection{Les risques des machines à pression}

La principale machine à pression sera la machine à vapeur alternative de Watt, qui va représenter dès le début du XIX ${ }^{e}$ siècle la principale source d'alimentation en énergie des installations industrielles. On compte ainsi en France un peu plus de 25000 machines en 1864, alors que leur nombre a atteint 40000 machines en 1875, et s'élève à 66500 en 1885 . Pendant les 10 premières années, le nombre d'explosions de machines double alors que celui-ci reste constant dans la période suivante. Le nombre d'explosions passe ainsi de 6,4 explosions pour 10000 machines en 1864 à 3,7 sur 10000 en 1885 . Les risques liés aux machines à pression, nouvelle technologie énergétique de l'époque, étaient déterminés par la maitrise de savoir-faire complexe aussi bien de pilotage par l'opérateur que d'entretien par l'utilisateur (CHAPUIS, 2003).

Comme pour les mines, la maitrise de la technologie au cours du temps, du fait de l'expérience et de l'échelle de mise en œuvre, a permis une relative sécurisation de l'usage industriel de cette technologie. De plus, cette expérience a été capitalisée, théorisée, et transformée en norme de fonctionnement donnant lieu à contrôle par une administration qui a étendu son activité à ce domaine : le service des mines.

La caractéristique des risques engendrés par les premières technologies industrielles est que, si elles font passer le risque à un niveau d'accidents récurrents, de grande échelle parfois catastrophique, ceuxci ont toujours des effets destructeurs confinés au site lui-même et limités à son personnel. Ces risques étant concentrés sur une activité technologique isolée, ses dysfonctionnements peuvent à terme être mis sous contrôle, par l'étude et la capitalisation d'expérience.

Des risques similaires se sont développés avec le développement de la vapeur sur l'activité ferroviaire dont les premiers et fréquents accidents ont fortement occupé les préoccupations sécuritaires de l'époque, il s'agit néanmoins déjà bien plus d'un avatar de l'accident de transport qu'une conséquence propre de la technologie vapeur.

Une nouvelle technologie énergétique liée au charbon, le gaz d'éclairage, va faire son apparition à la fin du siècle comme base énergétique des réseaux d'éclairages urbains. Il donnera lieu à de nombreuses controverses sur les dangers qu'il représente notamment dans les espaces urbains, alors que ses risques 
s'avèreront de fait assez limités. Ces activités technologiques commencent à générer des risques élargis qui émergent avec l'activité industrielle du deuxième système technologique.

\section{Le risque industriel du deuxième système industriel : la catastrophe technologique majeure}

Le deuxième système technologique, qui se met en place à partir des années 1880 , est fondé sur de nouvelles technologies génériques telles que la mécanique, l'électricité, la chimie et les énergies fossiles, notamment la dominance à partir des années 1940 des hydrocarbures.

Les industries d'assemblage qui se développeront à partir des technologies mécaniques en particulier n'engendreront pas de risques industriels catastrophiques. Ce sont les industries chimiques qui vont représenter le plus grand facteur de risque industriel, dans l'industrie des produits chimiques, comme dans sa composante des hydrocarbures.

\subsection{Les risques industriels des industries chimiques}

L'industrialisation de la chimie à partir des années 1880 , n'a pas permis une maîtrise technique qui rende ses process sûrs, de la production à la distribution. Ces process technologiques étaient amenés à manipuler des matières dont les réactions en particulier dans certaines conditions d'interaction et de concentration étaient particulièrement instables et génératrices d'explosions catastrophiques. Ceci, comme le montre la répétition sur la longue période d'accidents et de catastrophes, sans que l'accumulation d'expérience au cours du temps ait permis de mettre sous contrôle ces risques catastrophiques ${ }^{2}$.

C'est l'industrie des engrais et explosifs à base de nitrate d'ammonium qui illustre particulièrement cette situation. Le nitrate d'ammonium possède un comportement instable qui le conduit à des situations explosives en cas d'interaction avec de nombreux autres produits chimiques, mais aussi en réaction à une explosion voire à un choc électrique. L'analyse de la catastrophe majeure de l'usine AZF de Toulouse en septembre 2001, qui ne débouche sur aucune conclusion certaine, montre bien cette absence de maitrise. De fait, on recense plus d'une douzaine de catastrophes de ce type de 1921 à 2013, qu'elles aient lieu en usine, comme AZF mentionné ou une des toutes premières explosions en Allemagne en 1921 et la dernière connue dans une usine d'engrais au Texas en 2013 ; ou en situation de transport, particulièrement de manutention portuaire, comme la pire catastrophe industrielle des Etats-Unis à Texas City en 1947, explosion d'un cargo chargé de nitrate d'ammonium, qui fit 585 morts.

Le deuxième grand domaine de risques majeurs concerne un domaine périphérique de l'industrie chimique, qu'est l'industrie des hydrocarbures. Au moins une quarantaine de catastrophes et d'accidents majeurs depuis les années 1960 . Ces accidents d'hydrocarbure peuvent être des explosions de raffineries, terminaux et gazoduc ( $42 \%$ des accidents pétroliers), des naufrages, incendies et explosions de pétroliers ou trains de transport ( $43 \%$ des accidents pétroliers), et enfin des accidents, explosions et naufrages de plateformes off-shore. En terme de risques, les accidents de cette industrie apparaissent tenir moins aux risques intrinsèques de l'industrie, qu'aux aléas du transport et des risques des conditions maritimes, comme pour les plateformes, elle tient moins aux limites technologique de ses process, qu'aux conditions climatiques dans lesquelles cette matière dangereuse est transportée et parfois extraite dans le cas des plates-formes off-shore.

La seconde spécificité de cette industrie tient dans une conséquence majeure qui est surtout la pollution massive, par des produits chimiques toxiques, par des métaux lourds et surtout par des

2. Chronologie des catastrophes industrielles, consultable à l'adresse suivante :

https://fr.wikipedia.org/wiki/Chronologie_de_catastrophes_industrielles consultée le 14 septembre 2016.

๑ 2016 ISTE OpenScience - Published by ISTE Ltd. London, UK - openscience.fr

Page $\mid 6$ 
hydrocarbures, plusieurs millions de tonnes d'hydrocarbures ayant été répandus dans l'environnement par les accidents de l'industrie et du transport pétrolier.

Une autre catégorie majeure du risque chimique est l'explosion, incendie, destruction d'unités industrielles chimiques mais aussi l'incident technique qui conduit à relâcher massivement dans l'environnement des produits chimiques sous forme gazeuse ou liquide. Ce type d'accident a pu concerner une industrie apparemment anodine comme la minoterie, qui a entrainé en 1878 aux EtatsUnis, la destruction d'une usine par explosion de poussières de farines. Ce type d'accident va par la suite se multiplier aussi bien dans les usines que dans les silos.

Mais c'est dans les grandes usines chimiques que vont se produire des catastrophes majeures concentrées sur pratiquement 10 années : le $1^{\text {er }}$ juin 1974 en Grande-Bretagne : 28 employés trouvent la mort dans l'explosion qui détruit l'usine chimique de Flixborough (nord-est de l'Angleterre). L'incendie qui s'ensuit dure cinq jours et entraîne l'évacuation de 3.000 habitants des villages environnants. Le souffle de l'explosion a ravagé la région sur un rayon de plusieurs kilomètres. Outre les nombreux blessés, plus de 1800 habitations et près de 170 magasins sont endommagés.

Le 10 juillet 1976 c'est le célèbre accident de Seveso : la défaillance d'un des réacteurs de l'usine chimique du groupe chimique suisse Roche, au nord de Milan, provoque la propagation d'un nuage de produits chimiques, notamment de dioxine, dans l'environnement de quelque 36.000 personnes. Ce polluant contamine quatre communes voisines, dont Seveso, la plus touchée.

Au total, 736 personnes seront évacuées et 193 suivront des traitements médicaux. De nombreux animaux meurent, d'autres sont abattus par précaution. Par la suite, des dizaines de nouveau-nés ont présenté de graves malformations et de nombreuses femmes ont dû interrompre leur grossesse.

Le 3 décembre 1984 se produit à Bhopal en Inde l'une des pires catastrophes industrielles de l'histoire. Quelque 40 tonnes de gaz toxiques s'échappent de l'usine de pesticides de la société américaine Union Carbide, tuant 1750 personnes sur le coup. Quelque 2500 autres personnes trouvent la mort dans la semaine et, selon les associations de défense des victimes et de leurs familles, au moins 10.000 autres sont décédées par la suite. D'après des estimations officielles, plus de 500.000 habitants de Bhopal ont été affectés au total (GUSTIN, 2002).

Ces accidents chimiques, dus à des process complexes non maîtrisés, ont engendré des effets catastrophiques sur une large échelle, bien au-delà du site d'origine. Cela a entrainé la mise en place de dispositifs $^{3}$ de mise sous contrôle de ces risques combinant une réglementation, des procédures d'encadrement et de surveillance plus centrées sur l'aménagement des conditions externe de mise en œuvre de ces installation que d'une augmentation de la maittrise des process technologiques euxmême (POZZO, 2010).

\subsection{Les accidents nucléaires}

Sur une vingtaine d'incidents et d'accidents nucléaires recensés de 1952 à 2015 , trois seulement ont atteint des niveaux qui allaient au-delà des dommages limités au site. Ce sont les accidents de Three Mile Island aux Etats-Unis en 1979, celui de Tchernobyl en Union Soviétique en 1986 et celui de Fukushima au Japon en $2011^{4}$. Dans les deux premiers cas ces accidents sont attribuables à des erreurs de pilotage liées à la complexité du système à maîtriser. C'est le cas de Three Mile Island où le comportement du système devient brusquement incompréhensible aux opérateurs, du fait d'une insuffisance du système d'analyse, et d'un manque de discrimination du système homme-machine. (PIGNON, 1983). Il reste que l'accident de Three Mile Island n'aura pas de conséquence importante,

3. Directive Seveso, consultable à l'adresse suivante : https://fr.wikipedia.org/wiki/Directive_Seveso, consultée le 14 septembre 2016.

4. Cette partie s'appuie sur l'article «Liste des accidents nucléaires » accessible à l'adresse suivante :

https://fr.wikipedia.org/wiki/Liste_d\%27accidents_nucl\%C3\%A9aires consulté le 14 septembre 2016.

๑ 2016 ISTE OpenScience - Published by ISTE Ltd. London, UK - openscience.fr

Page $\mid 7$ 
une partie du cœur a fondu et un nuage à faible radioactivité a été relâché dans l'atmosphère. Mais cet accident aura des conséquences considérables en termes émotionnels notamment parce qu'il a inspiré le film à résonance considérable que sera Le syndrome chinois.

L'accident de Tchernobyl aura, à peu de choses près, une cause comparable, d'erreur de pilotage dans des conditions limites de fonctionnement du réacteur. L'accident s'est produit lors d'un exercice qui avait pour but de prouver que la centrale pouvait être relancée d'elle-même à la suite d'une perte totale du réseau électrique. Les réacteurs RBMK sont instables à faible puissance avec du combustible peu enrichi comme c'était le cas. Cet exercice a été conduit à une puissance trop faible et en plein pic xénon et iode : ce phénomène est qualifié d'« empoisonnement du réacteur ». La conduite à tenir à ce stade aurait été d'arrêter le réacteur pendant un à deux jours en maintenant un refroidissement permanent le temps que l'iode et le xénon se désintègrent naturellement.

Le réactif de l'explosion est le liquide caloporteur, en l'espèce de l'eau légère. La chaleur aurait provoqué la radiolyse de l'eau, puis la recombinaison de l'hydrogène et de l'oxygène libérés aurait provoqué l'explosion qui a soulevé la dalle de béton recouvrant le réacteur. Selon d'autres experts, l'explosion serait une explosion de vapeur, conduisant aux mêmes conséquences. Le graphite incandescent après l'explosion a fait fondre la gaine des crayons d'uranium, en zirconium et s'en est suivie la fusion de l'uranium lui-même qui dégagea des gaz et particules hautement radioactifs qui ont contribué à la contamination des nuages. L'incendie a été entretenu par la suite par la combustion du graphite. Il n'y a donc pas eu d'explosion nucléaire : si le point de départ est bien une réaction nucléaire en chaîne, c'est bien une réaction chimique ou une surpression de vapeur, et non une réaction nucléaire qui a provoqué la catastrophe. À la suite de l'accident, de grandes quantités de radio-isotopes radioactifs (et pour certains, extrêmement toxiques de surcroît), ont été libérées dans l'atmosphère. L'accident qui s'est produit à la centrale nucléaire de Tchernobyl dans le réacteur $\mathrm{n}^{\circ} 4$ est ainsi classé au niveau le plus élevé (le niveau 7) dans l'échelle INES qui mesure la gravité des accidents nucléaires.

Ses effets catastrophiques seront considérables, de 30 victimes à plusieurs milliers de morts selon les estimations, avec des effets de santé qui se perpétuent aujourd'hui encore. 300000 hectares rendus inhabitables, entrainant l'évacuation de 250000 personnes.

L'accident de Fukushima est d'une autre nature puisqu'il est d'abord la conséquence de très fortes catastrophes naturelles, un séisme d'intensité 9 et un tsunami d'une puissance en conséquence. Mais ces catastrophes naturelles n'ont pas entrainé la destruction de la centrale: "Les réacteurs se sont arrêtés comme prévu et sans dommage... l'origine de l'accident a été l'impossibilité dans laquelle se sont trouvé les responsables de faire face à la perte totale des moyens de refroidissement des combustibles usés, une fois la réaction nucléaire à l'arrêt, suite à l'inondation de la partie électrique et donc à l'insuffisance de la production d'électricité. » (BIGOT, 2011) Cette absence de refroidissement fait rapidement monter la température à $2500^{\circ} \mathrm{C}$ générant un nuage d'hydrogène dont l'explosion est à l'origine de la destruction de la centrale.

C'est donc la conjonction d'une configuration catastrophique naturelle et l'absence de réaction managériale pour mobiliser les moyens électriques de secours qui est à l'origine de la catastrophe. Deux cœurs de réacteurs sur 4 vont fondre, de larges émanations radioactives vont se répandre entrainant l'évacuation d'une importante population. L'accident de Fukushima sera classé à l'indice 7, indice maximal qui le situe au même niveau que Tchernobyl.

Dans tous les cas d'accidents nucléaires, c'est l'incapacité cognitive, de pilotage et de management à faire face à des situations inattendues et à des comportements imprévus qui en constituent l'origine. Dans les deux cas, les conséquences en sont catastrophiques. Par rapport aux occurrences d'accidents des technologies classiques, la manifestation de l'accident nucléaire est extrêmement rare. C'est une des caractéristiques de ce que l'on va désigner dans la période comme le risque technologique majeur. 


\subsection{La formalisation de ce type de risque, le risque technologique majeur}

Ces accidents récurrents, qui ont comme origine des dysfonctionnements majeurs des grandes industries, de transformation de la matière ou d'industries énergétiques, issues du deuxième système technologique, « industries du feu de la réaction chimique et de la pression », ont été formalisés à partir de la série concentrée de grands accidents chimiques ou nucléaires que nous avons décrite ci-dessus sous la notion de "risques technologiques majeurs» ou quand ce risque est avéré de "catastrophe technologique majeure » (LAGADEC, 1981).

La caractérisation de ces risques, consiste dans l'entrée du système technique dans une zone de comportement et de réaction, qui n'est pas comprise technologiquement et qui donne lieu à des réponses contre-productives, ou à une absence de réponse devant la soudaineté de la réaction générant l'accident. L'accident technologique majeur est le produit d'un défaut de maîtrise technologique devant un système atteignant des domaines limites incontrôlables, situation souvent combinée par des interactions avec des conditions inattendues qui amplifient le phénomène. Dans un système technique complexe peut se mettre en place une trajectoire aberrante, amplifiée par des boucles de rétroaction positives, dont l'interaction est inattendue et qui mène le système à la catastrophe. Cet inconnu ou inattendu conduit au désarroi des opérateurs et autorités, désarroi qui devient à son tour facteur d'amplification de la crise ou tout au moins de non-correction, et d'incapacité de mise sous contrôle.

La deuxième caractéristique de ce risque technologique majeur est son ampleur, contrairement aux accidents industriels du $1^{\mathrm{er}}$ siècle dont les effets se limitaient au site où avait eu lieu l'accident, et avec des victimes qui étaient exclusivement les opérateurs directs (l'accident minier ou l'accident d'usine traditionnel en étant les exemples), l'accident technologique majeur peut entrainer des destructions massives non seulement de l'installation à l'origine de l'accident, mais aussi des installations et habitations environnantes, il peut provoquer une mortalité massive, là aussi sur une large population environnante (Bhopal est un exemple du caractère extrêmement meurtrier de ce type d'accident), il peut interdire la vie sur des territoires considérables du fait des contaminations toxiques ou nucléaires. Et ceci, dans le cas du nucléaire en particulier, sur des périodes de temps considérables.

Il est, en tant que risque, très difficile à anticiper et à prévenir pour les raisons cognitives que nous avons dites et du fait de la complexité de son contexte. Si ces risques et types d'accidents sont toujours présents du fait de la pérennité de ces activités industrielles, il a émergé avec le système technologique actuel un nouveau type de risques d'une échelle plus large ou héritant de l'accumulation de nuisances de plus de deux siècles d'activité industrielle.

\section{Le système technologique contemporain : risque systémique et environnemental}

\subsection{La génération du risque systémique : société de l'information et société en réseau.}

Deux types de risques systémiques se sont développés : le premier, le risque électrique, est assis sur l'existence de réseaux de grande échelle, le second, basé aussi sur les réseaux, est le risque de l'accident informatique majeur voire de la cyber-catastrophe.

\subsubsection{Le risque électrique}

Une catégorie nouvelle de risque s'est manifestée par une série de catastrophes à partir des années 1960, il s'agite de la panne électrique massive. On en a recensé près d'une vingtaine jusqu'aux années 2000, elles ont particulièrement frappé l'Amérique du Nord et l'Europe, mais aussi plus récemment l'Inde, le Sénégal et l'Algérie. Ces crises du réseau électrique peuvent atteindre, du fait de l'ampleur 
des réseaux, un continent entier : nord-est des Etats-Unis et Ontario, 30 millions de personnes en 1965, France entière en 1978, Europe $2006^{5}$, à 670 millions d'usagers pour la panne indienne de juillet $2012^{6}$.

Ce risque majeur concerne la technologie électrique ancienne et bien maîtrisée, mais il se manifeste à ce degré de criticité par le niveau d'échelle atteint et surtout par l'accroissement de complexité et d'imbrication du réseau. Il tient aussi, dans l'importance de ces effets, à l'électrification universelle qui aura un maximum d'impact sur la société. Ces crises électriques manifestent la vulnérabilité des grands systèmes interreliés qui engendrent ce que l'on a pu appeler une « civilisation de la panne».

La grande panne électrique est en général provoquée par des courts circuits ou une surcharge du réseau, cette dernière ayant pour cause une saturation de capacités. Elle peut être due à une interaction critique avec des phénomènes météorologiques : séismes, ouragans, vagues de chaleur ou de froid extrême, raz-de-marée, inondations, etc. Cette défaillance majeure peut avoir pour origine des équipements en mauvais état liés à une insuffisance d'entretien et d'investissement et elle peut être amplifiée par des défaillances humaines et organisationnelles notamment dans la gestion et le pilotage du réseau.

Au-delà de la nuisance de la coupure de courant elle-même pour les usagers, celle-ci génère des arrêts et défaillances dans de nombreuses activités et services, parfois critiques, tels que l'arrêt de l'alimentation en eau potable : elle n'arrive plus dans les bâtiments, puisque les pompes ont besoin d'énergie pour fonctionner. Le chaos dans la circulation, privée de feux de la circulation, les ascenseurs, et tous moyens de transport électriques. Surtout elle provoque l'arrêt des dispositifs informatiques, ce qui entraîne des pertes économiques pour les entreprises, mais peut aussi entraîner de graves problèmes de sécurité.

La production du secteur industriel est gravement touchée. L'arrêt de la chaine du froid et des systèmes de chauffage par exemple peut produire des préjudices économiques dans les commerces, les grandes surfaces, les restaurants et les hôtels (les réfrigérateurs ne marchent pas).

\subsubsection{Le risque cybernétique majeur}

Le développement du système technologique contemporain est fondé sur la dominance d'une technologie générique de traitement de l'information, commencé dans la mutation technologique des années 1980, avec la dominance de ce que l'on a appelé la micro-électronique, relayée et amplifiée par la numérisation généralisée (BERRY, 2008), engendrant une convergence des systèmes techniques sous régulation numérique et la mise en réseau universelle (CASTELLS, 2001).

Ce nouveau système technique, support de la circulation de l'information, de la gestion du pilotage et de la régulation de la plus grande partie du système technologique et économique, va du fait même de cette position et de son caractère universel générer son propre régime de risque.

Le premier type de risque constaté est celui de la panne informatique. Celle-ci peut venir d'une défaillance matérielle - panne de matériel, incompatibilité entre composants. Ainsi, en 1996, la fusée Ariane 5, qui avait couté sept milliards de dollars et 10 ans de travail, s'est elle-même détruite, 36 secondes après son lancement. La raison : le système de bord a été incapable de convertir une donnée provenant d'un processeur de 64 bits dans un système de 32 bits. Le plus souvent la panne provient d'une défaillance logicielle : le « bug ».

Ce type de dysfonctionnement consubstantiel à la technologie informatique prend aujourd'hui une criticité croissante du fait de trois évolutions :

\footnotetext{
5. Panne généralisée documentée et analysé dans un rapport de l'UCTE, System disturbance, Bruxelles 2007.

6. Un descriptif documenté de l'ensemble de ces pannes électriques est consultable à l'adresse :

https://fr.wikipedia.org/wiki/Liste_des_pannes_de_courant_importantes. Consulté le 14 septembre 2016.

(c) 2016 ISTE OpenScience - Published by ISTE Ltd. London, UK - openscience.fr

Page $\mid 10$
} 
L'informatique étant au cœur du fonctionnement de la plupart des systèmes techniques, sa défaillance génère le blocage de la plupart de ceux-ci. La récente panne informatique de gestion des vols de la compagnie américaine Delta Airlines en août 2016 qui a bloqué ses vols pendant 8 heures en est une illustration. L'interconnexion généralisée, par les systèmes réseaux, peut généraliser une défaillance locale ou une saturation à tout ou partie du système ${ }^{7}$.

A ce risque par défaillance interne spontanée, se superpose le risque d'utilisation de cette défaillance par des acteurs mal intentionnés individuels, organisés, ou institutionnels. Les effets en sont l'intrusion, des destructions d'information et dégât possibles au système, prise de contrôle, pillage d'informations...

Ce risque informatique prend sa forme ultime avec le concept de cyberconflit (VENTRE, 2011). Cette nouvelle forme de confrontation, qui a connu ses premières manifestations lors de la guerre du Golfe en 1990, se généralise d'une manière endémique, amplifiant par le moteur de la confrontation, les risques du système cybernétique généralisé - le cyberespace est un multiplicateur de vulnérabilités.

\subsection{Le risque environnemental}

\subsubsection{Pollutions, prélèvements... un risque environnemental croissant}

Un type de risque est devenu majeur c'est la galaxie des risques environnementaux. Il s'agit de risques environnementaux directs comme les rejets et pollutions et les prélèvements excessifs sur la ressource, issus d'activités industrielles portées par des technologies destructrices de l'environnement, mais qui prennent une dimension particulière aujourd'hui, par l'échelle à laquelle ils se concrétisent. Ainsi la pollution qui s'est longtemps limitée à la pollution proprement toxique s'est élargie à la pollution par accumulation: tout rejet de quelque matière que ce soit, constitue une agression destructrice de l'environnement lorsqu'il est relâché en grande quantité. Le cas des accumulations de lisier de porc en rejet de l'élevage intensif, comme de la constitution de ce que l'on appelle le $« 7^{\mathrm{e}}$ continent » plastique en sont des illustrations significatives.

Il en est de même pour le prélèvement de ressources naturelles et minières. Cette modélisation de l'excès de prélèvements de ressources naturelles par le système économique en place, et en croissance, avait déjà été menée dans les années 1970 et elle avait montré que le type de système technologique qui avait alimenté la croissance industrielle et notamment celle d'après-guerre n'était pas soutenable (MEADOWS 1972).

Ces prélèvements excessifs concernent particulièrement l'énergie et en particulier le pétrole dont la rupture d'approvisionnement voire le déclin de la production constituerait, au-delà des problèmes environnementaux, un risque supplémentaire de perturbation profonde du fonctionnement du système technico-économique comme l'a montré la crise de 1973, et comme le laissent craindre les poussées de hausse du prix du pétrole (DOLIQUE, 2009). Au-delà de l'énergie, l'ensemble des prélèvements y compris de ressources renouvelables a atteint un niveau tel que les calculs montrent que les prélèvements de ressources sur la terre en 2016, vont au-delà des quantités correspondant à son rythme de reproduction dès le mois de septembre, et que cette date a avancé régulièrement ces dernières années.

\subsubsection{Vers le risque environnemental global}

Mais le risque environnemental n'a plus seulement une action destructrice instantanée, mais un changement de nature, un saut qualitatif engendré par l'accumulation de pollution, sur l'ensemble de la période du développement industriel engendre aujourd'hui un risque environnemental global et dynamique. Risque global puisqu'il met en œuvre des phénomènes concernant l'environnement global,

7. « Le bug de l'an 2000 », qui ne s'est heureusement pas concrétisé, représente une modélisation anticipative de ce type de collapsus. 
risque dynamique au sens où ce sont les mécanismes de régulation de la biosphère qui sont altérés, déclenchant eux même des mécanismes d'amplification. L'élément fondamental de cette régulation, le $\mathrm{CO}^{2}$ dans l'atmosphère, base de fonctionnement de l'effet de serre, avait été régulé sur le long terme par sa captation et son stockage dans la biomasse fossilisée, «Depuis la domestication des énergies fossiles et le passage à une économie du carbone, il a fallu très peu de temps à l'homme pour disperser cette matière et l'énergie qu'elle comprenait. La biosphère se trouve aujourd'hui incapable d'absorber toute la quantité de $\mathrm{CO}^{2}$ rejetée dans l'atmosphère » (DOLIQUE, 2009, p. 216).

Le risque global majeur principal actuel est le réchauffement global car pour la première fois il a un impact sur l'ensemble de la biosphère et risque non seulement de dégrader les conditions de vie et de développement de l'humanité mais peut, à terme, mettre en cause ses conditions mêmes de survie.

\section{Conclusion}

Cette présentation montre que chacun des trois systèmes technologiques successifs qui ont animé la dynamique de développement mondial a généré des types de risques qui leur étaient spécifiques. Il apparaît aussi, qu'au cours de ce processus les risques les plus anciens ne disparaissent pas mais, comme dans le cas des risques miniers ou des grands risques chimiques, se déplacent géographiquement. Selon l'évolution des systèmes technologiques les risques ne se remplacent pas, mais partiellement s'accumulent.

Pour les plus récents, la numérisation généralisée et l'interconnexion universelle en élargissent considérablement l'échelle d'impacts et d'interaction. De plus on voit apparaitre un nouveau type et une nouvelle échelle de risques, la dégradation globale de la biosphère, par accumulation de nuisances faisant émerger un risque global qui est lié à des technologies particulières mais à l'accumulation de leurs nuisances sur la longue durée.

Ce risque global fait apparaitre une limite qui n'avait pas été prise en compte dans l'analyse technologique et industrielle passée c'est la possibilité d'une contradiction globale du système technologique mondial avec l'environnement planétaire par un phénomène entropique (DEMYNCK, 1980).

C'est cette globalité qui oblige à penser le développement contemporain comme celui d'une société du risque (BECK, 1992) dans laquelle le risque devient une variable dominante de l'évolution du système technologique, de sa configuration interne par le blocage de certaines technologies particulières devenues impraticables. Un nouveau paradigme élargi du système technologique dans son environnement sociétal et écologique en voie d'élaboration (DE WECK, 2011), pourrait permettre de penser les nouvelles trajectoires d'innovation (DIEMER, 2012).

Cette mutation entraine une question : la prise en compte des risques majeurs et en particulier du risque environnemental global nécessite-t-elle un changement de système technologique, ou entrainerat-elle une mise en cohérence du système technologique contemporain autour de se technologies « néguentropiques », technologies de l'information, biotechnologies, énergies renouvelables, en abandonnant les anciennes technologies «carbonées 》 perpétuées des systèmes technologiques anciens?

\section{Bibliographie}

AÏT-EL-HADJ S., «Eléments de modélisation systémique de la dynamique technologique », p. 99-122, Marché et organisations, $\mathrm{n}^{\circ} 23$, l'économie du changement, 2014. 
AÏT-EL-HADJ S., « De la théorie du système technique à la systémique technologique, une formalisation pertinente pour rendre compte de l'innovation technologique». p. 227-250, Innovations, Revue d'économie et de management de l'innovation, $\mathrm{n}^{\circ}$ 46, vol. 1, 2015.

AMOUDRU C., POT F., « Sécurité et catastrophes minières », p. 175-187, Culture Technique nº 11, septembre, 1983.

BECK U., Risk Society: Towards a New Modernity. Sage, New Delhi, 1992.

BERRY G., Pourquoi et comment le monde devient numérique, Collège de France, Fayard, Paris, 2008.

BIGOT B., «L'énergie nucléaire après Fukushima », p. 123-128, Futuribles n 376, 2011.

Chaire de cyberdéfense et de cyber sécurité, Géopolitique du cyberespace, Diplomatie, grands dossiers n 23, 2014.

CASTELLS M., La galaxie internet, Fayard, Paris, 2001.

CHAPUIS C., «Risque et sécurité des machines à vapeur au XIX siècle », p.203-17, Culture Technique ${ }^{\circ}{ }^{\mathrm{e}} 11$, septembre, 1983.

DAUPHINE A., PROVITOLO D., Risques et catastrophes - Observer, spatialiser, comprendre, gérer, Armand Colin, Paris, 2013.

De WECK O., Ross D., Magee C. L., Engineering Systems. Meeting Human needs in a complex technological world, The MIT Press, Cambridge, Massachusetts, 2011.

DIEMER A., « La technologie au cœur du développement durable, mythe ou réalité », Innovations, cahier d'économie de l'innovation, p. 73-92, n 37, 2012.

DOSI G., Technological paradigms and technological trajectories, a suggested interpretation of the determinants and directions of technical change, p. 147-162, Research Policy, 11, North Holland publishing, 1982.

FRESSOZ J. B. L'apocalypse joyeuse. Une histoire du risque technologique, Le Seuil, Paris, 2012.

GILLE B., «Prolégomènes à une histoire des techniques », dans Histoire des techniques, p. 4-118, La Pléiade, Paris, 1978.

GUSTIN J. L., "Leçons des accidents majeurs dans l'industrie chimique », Les techniques de l'ingénieur, Volume Management de la sécurité, p. 229-243, 2002.

HUGHES Th. P., The evolution of large technological systems, in the Social construction of technological systems, p. 5182, edited by E. Bijker, Th. P. Hughes, T. Pinch, MIT Press, Cambridge, Massachusetts, 1987.

KAHANE, B., Les nanotechnologies entre fiction et réalité, p. 5-20, Futuribles, nº 384, avril, 2012.

LAGADEC P., Le risque technologique majeur, Politique, risque et processus de développement, Pergamon, Paris, 1981.

LANDES D., L'Europe technicienne ou le Prométhée libéré. Révolution technique et libre essor industriel en Europe occidentale de 1750 à nos jours, NRF Gallimard, Paris, 1975.

LE ROUX T., «Accidents industriels et régulation des risques : L'explosion de la poudrerie de Grenelle en 1794 », Revue d'histoire moderne et contemporaine, Paris, Belin, n 58-3, juillet-septembre, p. 34-62, 2011.

MEADOWS D., RANDERS J., BEHRENS W., The Limits to Growth, Universe Book, New-York, 1972.

MENSCH G., Stalemate in Technology: innovations Overcome the depression, Ballinger, Cambridge, Massachusetts, 1980.

NEIRYNCK J., Le huitième jour de la création, introduction à l'entropologie, Presses polytechniques romandes, 1986.

PIGNON D., "Pathologie d'un système technologique hyper complexe, la crise de Three Mile Island», Culture Technique $\mathrm{n}^{\circ} 11$ septembre, p. 79-91, 1983.

PORTNOFF A.Y., «L'hyperchoix des matériaux, dans La révolution de l'intelligence », p. 23-39, numéro spécial, Sciences et techniques, $\mathrm{n}^{\circ}$ 97, octobre, 1983.

POZZO B., The Implementation of Seveso Directives in an Enlarged Europe: A Look into the Past and a Challenge for the Future, Kluwer Law International, 2010.

SOUCHON P., De la prévention des risques à l'ingénierie de la sécurité, thèse de sciences de gestion, université Jean Moulin Lyon 3, 2010.

VENTRE D., Cyberespace et acteurs du cyberconflit, Hermès-Lavoisier, Paris, 2011. 